\title{
La place de la grammaire dans l'enseignement du français langue étrangère dans le cycle secondaire en Algérie
}

\begin{abstract}
:
In this article, we have brought to light the place reserved to grammar in the FLE teaching in the secondary cycle. In effect, we analyzed guides, accompanying documents, programs and manuals of the three levels what allowed us to make a number of remarks. These bring us to say that grammatical difficulties facing the learners are partly due to the tiny place that occupies grammar in the secondary cycle and to the multiple theories that underlie it.

Key-words: grammar, didactic of the FLE grammar, linguistic theories, grammatical difficulties
\end{abstract}

Hammouche Sarra

Faculté des lettres et des langues

Département de français

ENS Constantine

Algérie

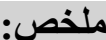

في هذا المقال، قمنا بتسليط الضوء على المكان المخصص ،

للنحو في تعليم لغة أجنبية في الطور الثانوي. في هذا

الثأن، قمنا بتحليل الأدلة و الوثائق المرفقة و البرامج

بالإضافة إلى الكتب المدرسية للأطوار الثلاثة ما مكنا من

تقديم بعد الملاحظات. تبين لنا هذه الأخيرة أن نقول أن

الصعوبات النحوية التي يتعرض لها المتعلمين ترجع جزيئا

إلى المكان الضيق للنحو في الطور الثانوي و تعدد

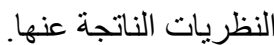

الكلمات المفتاحية :النحو ، تعليمية النحو لمادة الفرنسية

لغة أجنبية، النظريات اللسانية،الصعوبات النحوية

\section{Introduction :}

Tout au long de notre expérience à l'ENS, lors de nos déplacements avec les stagiaires dans les lycées, nous avons constaté que dans la réalité scolaire en Algérie, les apprenants de $3^{\text {ème }}$ AS ont beaucoup de difficultés à s'exprimer en langue française. Celle-ci est riche et pleine de subtilités en tout genre, notamment en grammaire qui est au centre de l'apprentissage d'une langue. 
Ainsi, Marie Eve Damar (2009 :60) affirme : "La grammaire au sens où nous l'entendons joue un rôle partout en classe de langue, puisqu'elle renvoie à la construction de tout énoncé. Ce n'est donc pas "un outil linguistique » parmi d'autres. "

L'enseignement de la grammaire est donc indispensable et essentiel dans l'apprentissage du FLE puisqu'il est important de respecter les normes d'une langue pour bien communiquer et se faire comprendre.

Dans notre article, nous cherchons à situer la place qu'occupe la grammaire dans l'enseignement du FLE en Algérie précisément dans les manuels scolaires du cycle secondaire. C'est pourquoi, nous nous interrogeons sur les méthodes et les courants linguistiques adoptés dans l'enseignement de la grammaire au lycée.

Nous émettons l'hypothèse que la place de la grammaire n'est pas importante au secondaire. Pour confirmer ou infirmer cette assertion, notre étude portera sur un corpus formé des manuels scolaires, des consignes données dans les documents d'accompagnement et les guides de l'enseignant. Notre analyse aura pour objet la place de la grammaire dans ces documents.

Nous nous appuierons sur les méthodes d'enseignement de la grammaire du FLE et les théories linguistiques qui les sous-tendent

Notre démarche consistera à analyser en premier lieu les directives concernant la grammaire dans les documents d'accompagnement et les guides de l'enseignant, puis à examiner les exercices de grammaire des manuels en précisant leurs types et les théories linguistiques auxquelles ils se référent.

Même si dans les documents d'accompagnement, les auteurs donnent souvent la théorie linguistique qui les a inspirés, l'analyse des manuels permettra de vérifier concrètement à quelles théories ils font référence.

Acceptions du mot " grammaire "

Avant de passer à l'analyse, il faudrait sans doute donner le sens du mot « grammaire ».

Selon Jean-pierre Cuq (1996: 7), le terme de grammaire vient du mot grec « gramma » qui signifie la lettre. Il souligne que contrairement au mot et à la notion de linguistique qui ne datent que du $19^{\text {ème }}$ siècle, la grammaire comprise au sens de réflexion sur le langage est l'une des plus anciennes tentatives de connaissance de l'homme. Le terme de grammaire est très ambiguë et délicat à définir parce qu'il est polémique.

Selon Jean-pierre Robert (2002: 84) : la grammaire est" l'étude scientifique des énoncés d'une langue à travers leurs structures morphologiques et syntaxiques »

Jean Dubois, Mathée Giacomo, LouisGuespin et al (1973: 238 ) définissent le terme de grammaire comme la description complète de la langue, c'est-à-dire des principes d'organisation de la langue. 


\section{La place de la grammaire dans l'enseignement du français langue}

étrangère dans le cycle secondaire en Algérie

Robert Galisson et Daniel Coste (1976 : 253), eux, définissent la grammaire comme " une description de la morphologie et de la syntaxe d'une langue » La grammaire est donc l'ensemble des normes qu'il faudrait connaitre et maîtriser pour bien communiquer.

Voilà pourquoi, nous voudrions dans cet article mettre en évidence la place réservée à la grammaire dans l'enseignement du FLE dans le cycle secondaire en Algérie.

La grammaire dans les guides, les documents d'accompagnement et les programmes

Dans le guide de 1 ère AS, il est mentionné que "les exercices (de grammaire) ne sont pas à faire dans l'ordre proposé dans le manuel mais doivent s'insérer dans la progression établie par l'enseignant» (p. 8). Ces exercices "ont été choisis en fonction des faits de langue dégagés dans les questionnaires de lecture analytique donc liées au programme » (p.8).

Il est indiqué aussi qu'il faut "proposer des exercices et des séances de grammaire aux élèves en difficulté et de remédier aux lacunes antérieures 》 (p.8).

Dans le document d'accompagnement de $1^{\text {er }}$ AS, pour chaque projet, on propose au niveau phrastique des points de grammaire à rappeler à travers des exercices: la subordonnée relative, la subordonnée d'opposition, les subordonnées de cause et de conséquence, les tournures impersonnelles, la voix passive pour le projet «La vulgarisation de l'information scientifique » (p.38), l'interrogation partielle, l'injonction, les marques personnelles pour le projet «L'interview»( $\mathrm{p} .40)$, les subordonnées concessives, les différences entre « parce que » et « puisque », le conditionnel pour le projet « la lettre ouverte» (p.42), la nominalisation, le passé composé, le plus que parfait, les verbes introducteurs, les connecteurs pour le projet «Le fait divers » (p.43), le passé simple, le conditionnel, la conséquence, les verbes introducteurs et les verbes de perception pour le projet « La nouvelle » (p.45).

Dans les documents d'accompagnement et le guide de $2^{\text {ème }} \mathrm{AS}$, on peut lire certaines orientations à propos de l'apprentissage de la grammaire.

Ainsi, dans le document d'accompagnement, les auteurs précisent que « chaque séance intégrera les moments de manipulations syntaxiques et lexicales » (p. 4). Il est dit aussi que " des moments de la classe seront consacrés à la correction des exercices. Ces derniers doivent installer des automatismes nécessaires à l'atteinte d'un certain degré de maîtrise du code. ».

On peut lire aussi dans un paragraphe intitulé " Comment aborder la grammaire au secondaire " (p. 23) que "le travail de la grammaire au secondaire ne constitue pas un objectif en soi. La grammaire est à mettre au service de la compréhension du texte et de la pratique effective de la langue. » L'apprenant devra prendre conscience de la matérialité de la langue. C'est-àdire, il accordera plus d'intérêt aux choix linguistiques opérés par un auteur et 
aux phénomènes linguistiques en relation avec l'intention d'énonciation. On est dans une grammaire énonciative. Celle-ci étudie la langue, et les énonciateurs la prennent en charge en liant la forme au sens car on associe la langue à son utilisation et les mots grammaticaux ont une signification à travers le moment de la production de l'énoncé.

Marie-Françoise Narcy-Combes (2005 :70) affirme : " La linguistique énonciative travaille sur le discours comme processus mental de construction de sens. "

Dans le guide de $2^{\text {ème }}$ AS (p.17), on demande à ce que "Les activités d'acquisition des outils linguistiques (lexique, syntaxe, conjugaison, orthographe)" soient " intégrées dans les apprentissages fondamentaux (compréhension et expression) en fonction des besoins des apprenants et des objectifs de la séquence. Elles sont reliées à des situations concrètes de communication et doivent être considérées comme des réponses à des besoins déterminés à partir des activités de compréhension et d'expression » (p.17). Ce sont des compétences de communication.

Dans le guide de $3^{\text {ème }} \mathrm{AS}$, les auteurs précisent que les activités proposées (dans le manuel) prennent en charge les différents domaines d'apprentissage (écrit et oral) pour l'installation (ou le développement) d'une compétence de communication (p.32). Les auteurs ajoutent qu'il s'agit de mener l'élève vers une maîtrise progressive des mécanismes et des structures de la langue et renforcer les capacités à interroger les éléments linguistiques contenus dans un discours pour déceler l'intention communicative du locuteur (ou du scripteur) en tant que destinataire d'un discours d'une part, et rendre perceptible son intention communicative en tant que producteur de discours, d'autre part (p.36). C'est ainsi que les verbes introduisant l'interpellation doivent tenir compte du statut de l'émetteur et du récepteur de l'appel (p.46).

Dans le document d'accompagnement de $3^{\text {ème }} \mathrm{AS}$ à la page 70 , quelques remarques sont faites sur l'emploi des temps. Dans le texte historique, l'imparfait, le passé simple correspondent à la distance temporelle qui sépare le présent de l'écriture des événements relatés (Michelet). «Le choix du présent de narration actualise les événements rapportés et fait du lecteur le témoin d'une histoire en train de se construire». (Manceron).

Il est dit aussi que «le présent intemporel est le temps habituel du texte argumentatif puisque l'argument est valable de façon générale. » (p.72).

Par conséquent, toutes ces indications insistent sur le fait que la grammaire au secondaire doit être enseignée d'une manière ponctuelle afin d'éclairer la compréhension et faciliter la production écrite.

Nous avons également pris connaissance des programmes officiels dans le cycle secondaire (répartitions annuelles distribuées par l'I.E.S de la wilaya de Constantine) et nous avons constaté qu'il est demandé de clarifier un certain nombre de points de langue en relation avec l'objectif du projet et de la 


\section{La place de la grammaire dans l'enseignement du français langue étrangère dans le cycle secondaire en Algérie}

séquence étudiée par exemple le présent intemporel est étudié dans le cadre de l'exposé, le discours rapporté est étudié dans les témoignages des textes historiques.

\section{La grammaire dans les manuels}

Dans les manuels du secondaire, il n'existe aucune leçon explicite de grammaire. Il n'y a que des exercices de grammaire dans les manuels de $1^{\text {ère }}$ AS et $3^{\text {ème }}$ AS. Dans celui de $2^{\text {ème }} \mathrm{AS}$, nous avons quelques rappels sous forme d'exemples, de définitions ou de tableaux avant les exercices proposés.

Ainsi, des encadrés «Retenir» sont présentés pour les points de grammaire suivants : les progressions à thèmes dérivés, les relations logiques explicites, les relations logiques implicites, la comparaison et la métaphore, la subordonnée relative, les substituts, le discours direct, la caractérisation.

Par exemple, pour ce dernier point grammatical. Voici le contenu de l'encadré (p.188) qui lui est réservé :

« Pour caractériser, on peut utiliser :

- L'adjectif : une jolie maisonnette avec un petit jardin.

- Le nom : La fille de Scamboli

- La proposition relative : un nez magique qui grandira à chacun de ses mensonges.

Caractériser une personne, un lieu ou un objet veut dire apporter des informations supplémentaires pour mieux présenter, situer, préciser ou décrire cette personne, ce lieu ou cet objet. »

Il faut remarquer que les exercices à la fin de chaque projet correspondent aux points de langue mentionnés dans les programmes et concernent aussi bien la grammaire que le lexique.

Nous nous sommes donc attelée à examiner les exercices et à déceler ceux qui correspondent aux points de grammaire proposés dans les programmes, de donner le type d'exercice et le lien qu'il a avec l'objectif du projet et de la séquence étudiée ainsi que la théorie linguistique utilisée.

\section{Dans le manuel de $\mathbf{1}^{\text {ère }} \mathrm{AS}$}

Dans le manuel de $1^{\text {ère }}$ année secondaire, il y a deux parties dans le projet 1 "Campagne d'information". La première partie a comme intention communicative "l'exposé". Les points de grammaire proposés ont un lien avec cet objectif.

Ainsi, les tournures impersonnelles sont étudiées dans l'exercice 5 page 64 où l'on demande de transformer des phrases pour obtenir des constructions impersonnelles. Par exemple, pour la phrase «Des milliers d'années se sont passées avant que les hommes ne domestiquent la nature.» On attend de l'apprenant la transformation suivante : «Il s'est passé des milliers d'années avant que les hommes ne domestiquent la nature. » 
Un exercice d'identification $\mathrm{n}^{\circ} 8$ page 65 concerne la relative. L'apprenant doit reconnaitre la relative déterminative : la $3^{\text {ème }}$ phrase par exemple "L'eau qui n'est pas traitée est à l'origine de nombreuses maladies. » et la relative explicative comme la $6^{\mathrm{ème}}$ phrase «Les déchets industriels qui sont de plus en plus importants sont des poisons dangereux. »

L'élève doit ajouter une virgule avant le pronom relatif «qui » et une autre après le mot «importants » pour mettre en évidence qu'il s'agit d'une relative explicative.

Les exercices, 6 et 7, pages 64 et 65 , exigent des transformations respectivement à la voix active et à la voix passive. Tous ces exercices se référent à la grammaire transformationnelle puisqu'il s'agit d'apporter des transformations aux phrases proposées.

L'exercice 3 page 63 est un exercice de complétion où il faut compléter les phrases par des conjonctions de coordination convenables.

Dans la phrase : «Les hommes détruisent l'habitat d'une multitude d'animaux lorsqu'ils abattent des forêts entières ...une fois leur habitat détruit, ces animaux ne peuvent plus survivre », l'apprenant doit ajouter la conjonction de coordination « donc».

D'autres phrases à relier sont proposées dans l'exercice 4 page 63 pour mettre en relief les rapports logiques de cause et de conséquence.

Ainsi, pour les phrases « Les terres désertiques manquent d'eau et contiennent souvent trop de sel. Les plantes y sont rares », l'apprenant les relira par «c'est pourquoi ».

Ces deux derniers exercices mettent en relief les rapports logiques entre les phrases et font référence à la grammaire textuelle.

Dans la $2^{\text {ème }}$ partie du projet 1 «L'interview », les points de grammaire sont en relation avec l'intention communicative puisqu'on étudie l'impératif et l'indicatif dans l'exercice 11 page 96 où on demande de mettre les verbes entre parenthèses aux modes et temps convenables. L'interrogation est abordée dans les exercices 6 et 8 page 94. Dans l'exercice 6, il est demandé s'il s'agit d'une vraie question: "Comment avez-vous appris cela?», une fausse question: «Qui d'autre que ce grand savant aurait pu faire cette découverte? » ou une question pour atténuer un ordre «Ne pourriez-vous pas baisser le son de la radio ?»

Dans l'exercice 7, l'apprenant doit compléter les phrases interrogatives par des pronoms interrogatifs. Ainsi, pour les phrases :

«- ........pensez-vous faire pendant les vacances?

- Je compte visiter l'Egypte et la Syrie. », l'élève doit ajouter le pronom interrogatif "Que». Dans ces exercices, il s'agit de grammaire énonciative puisqu'on se réfère au sens du texte.

Dans les exercices 12 et 13 page 96, il s'agit de grammaire transformationnelle et énonciative. On demande à l'apprenant de transformer des textes : écrire un 


\section{La place de la grammaire dans l'enseignement du français langue étrangère dans le cycle secondaire en Algérie}

dialogue entre deux amis au style direct à partir d'un texte au style indirect dans l'exercice 12 et retrouver les répliques des interlocuteurs à partir d'un texte de Maurice Leblanc dans l'exercice 13.

Le projet 2 《Rédiger une lettre ouverte» a pour intention communicative l'argumentation et les points grammaticaux étudiés sont les articulateurs comme par exemple: parce que, mais, puisque, par conséquent, bien que, malgré...

Dans l'exercice 8 page120, l'élève doit trouver l'articulateur «malgré » pour compléter la phrase :

«...........son prix élevé, l'ordinateur est un outil de travail indispensable. »

Dans l'exercice 7 page 120, il est demandé à l'apprenant de compléter un dialogue par des modalisateurs proposés : sûrement, peut-être, franchement, malheureusement, sans-doute.

Ainsi, il doit mettre "malheureusement» pour compléter la phrase «.........., je ne sais pas où le joindre à cette heure-ci. »

Sous forme d'exercices de complétion, il s'agit de grammaire textuelle pour l'exercice 8 et de grammaire énonciative pour l'exercice 7 .

Le projet 3 «Ecrire une biographie » a deux intentions communicatives.

Dans la première partie, il s'agit de relater un fait réel, un fait divers. La transformation passive et la nominalisation sont étudiées à travers l'exercice 1 p.144, 145, 146. L'élève doit par exemple mettre le verbe "prononcer» à la voix passive dans le troisième fait divers «La réclusion à perpétuité a été prononcée samedi par le tribunal criminel d'Oran... »

Il doit trouver aussi pour chaque fait divers un titre sous forme de phrase nominale : «Arrestation de deux policiers espagnols pour vol. » pour le fait divers $n^{\circ} 4$. Il s'agit dans cet exercice de grammaire transformationnelle : transformation passive et nominalisation d'une phrase.

Dans la seconde partie, il est demandé de relater un fait fictif.

Dans les exercices 1, 2, 3 et 4 p.189, l'élève doit mettre les verbes aux temps convenables.

Dans les exercices 5 et 8 page 190, il doit faire attention à la concordance des temps.

Tous ces exercices abordent les temps du récit (présent, imparfait, passé simple, plus que parfait) : il s'agit donc de grammaire textuelle.

\section{Dans le manuel de $2^{\text {ème }}$ AS}

Dans le manuel de deuxième année secondaire, le projet 1 «Réalisation d'un dossier documentaire »a pour intention communicative de présenter et exposer un fait. On étudie par conséquent les progressions thématiques dans l'exercice page 38 où l'on doit retrouver le schéma de progression à thèmes dérivés dans un petit texte donné : "Dans un projecteur, quand une photo du film est projetée, la pellicule reste fixe. La pellicule avance ensuite d'un cran. Pendant ce mouvement un obturateur vient boucher l'objectif... » 
Dans l'exercice 1 page 36, l'apprenant doit relever les présentatifs « c'est », par exemple dans la phrase "Le laser, c'est un pinceau de lumière intense colorée. »

Dans l'exercice 2 page 36, il doit réemployer les présentatifs « il y a, il existe, il s'agit de, c'est, voici » en répondant à une liste de questions proposées.

$\mathrm{Ce}$ sont les exercices d'identification, de classement, de réemploi. Il s'agit de grammaire textuelle et il en est de même pour l'exercice page 46 portant sur le gérondif et les exercices 1 et 2 page 47 portant sur le conditionnel.

Dans les exercices 1 et 2 page 42, on étudie les rapports logiques de cause et de conséquence. L'apprenant doit répondre à une série de questions en utilisant le rapport logique de cause puis le rapport logique de conséquence. Ainsi, à la question «Pourquoi vieillit-on?» On pourrait donner les réponses suivantes : «L'homme vieillit parce que ses cellules se dégradent. » et «Les cellules de l'Homme se dégradent si bien qu'il vieillit.» Il s'agit de grammaire énonciative.

Le projet 2 «Mettre en scène un spectacle» en relation avec l'intention communicative «dialoguer pour raconter» prévoit l'étude des tournures interrogatives et exclamatives dans l'exercice p.180 où on demande de relever les tournures exclamatives qui indiquent l'hésitation, l'étonnement, l'interjection ou la satisfaction. Par exemple : dans l'extrait de la pièce de théâtre de Jean Tardieu, on peut relever des tournures exclamatives indiquant l'hésitation (Bien...oui dame !), l'étonnement (Là !), l'interjection (Ouf!), la satisfaction (Bravo ! bravo !). Il s'agit de grammaire énonciative.

Dans les exercices 1 et 2 page 187, il est demandé de transformer deux textes du style indirect au style direct: par exemple, la $1^{\text {ere }}$ phrase du $1^{\text {er }}$ texte (Le domestique déclara que, depuis un mois, son maître semblait agité.) doit être transformée à la voix active (Le domestique déclara : «Depuis un mois, mon maître semble agité. »). Ces exercices se réfèrent à la grammaire transformationnelle.

Enfin, les exercices 1, 2 et 3 page 188 et 189 portent sur la caractérisation. Celle-ci se fait avec un adjectif, un complément de nom ou une subordonnée relative et elle permet la description des personnages. Dans l'exercice 2, on demande à l'apprenant de relever la caractérisation de Pinocchio (texte p.188) avec un adjectif " nez magique », avec un nom «Le don d'un nez », avec une subordonnée relative "un nez qui grandira à chacun de ses mensonges ». Il s'agit de grammaire textuelle.

Le projet 3 «Procès pour défendre des valeurs humaines »a pour intention d'argumenter avec des relations logiques. Celles-ci peuvent être explicites dans les exercices 1, 2, 3 p.73 qui se référent à la grammaire textuelle avec des exercices d'identification (l'exercice 1 propose d'entourer les connecteurs logiques et de nommer les relations logiques dans un petit texte) ou de 


\section{La place de la grammaire dans l'enseignement du français langue étrangère dans le cycle secondaire en Algérie}

complétion (l'exercice 3 invite l'apprenant à compléter des phrases en exprimant la cause, la conséquence et l'opposition)

Les relations logiques peuvent être aussi implicites dans l'exercice p. 75 et on se réfère alors à la grammaire transformationnelle puisqu'on demande à l'apprenant de transformer des rapports logiques implicites en rapports logiques explicites. Par exemple, l'élève doit transformer la phrase « Opération prestigieuse pour les Etats-Unis, la conquête de la Lune se révéla très coûteuse. » en phrase avec rapport logique explicite.

Le projet 4 «Présenter le lycée de vos rêves » a pour intention communicative « relater pour informer » et nous retrouvons la grammaire textuelle à travers les exercices p.141, 142 et 143 portant sur la comparaison et la subordonnée relative.

Dans le manuel de $3^{\text {ème }}$ AS

Dans le manuel de troisième année secondaire, le projet 1 «Dans le cadre de la commémoration d'une journée historique, réaliser une recherche historique », l'objet d'étude porte sur des textes et documents d'histoire. Les points de grammaire proposés sont la nominalisation, dans l'exercice 3 p.61, pour parler d'un événement. Il s'agit de grammaire transformationnelle puisque les élèves doivent transformer sur le modèle donné : «Deux cargos sont entrés en collision : collision de deux cargos.

Les exercices 1 p.61 et 4 p.62 proposent soit de compléter par des indices temporels (puis, d'abord, enfin, lorsque, pendant que, avant que) soit de relever les indices temporels qui sont en relation avec la chronologie des événements. On se réfère à la grammaire énonciative.

Le projet 2 "Organiser un débat d'idées » propose des exercices sur la concession.

Des exercices d'identification 1, 2 p.116 et des exercices de complétion 3 p.116 et 5 p.117 sont en rapport avec le débat d'idées opposées. On demande respectivement de relever les connecteurs, de donner leurs valeurs, de trouver le connecteur (et, ainsi, ou, d'abord, mais, et puis, pourtant, parce que) correspondant à la valeur argumentative donnée (cause, addition, opposition...) ou encore de compléter un texte par des connecteurs donnés dans une liste (c'est pourquoi, pourtant, en effet, mais, donc, de plus, puisque, car). Il s'agit bien sûr de grammaire textuelle.

Dans le projet 3 «Mobilisation autour des causes humanitaires» en rapport avec l'appel, l'exercice 9 p.170 permet d'étudier les différentes formes d'injonction. L'apprenant doit compléter des petits textes par des verbes choisis dans une liste de verbes performatifs (appeler, demander, prier, conseiller, ordonner, exhorter). Il s'agit donc de grammaire énonciative et pragmatique.

Enfin, dans le projet 4 "Rédiger une nouvelle fantastique », un exercice de complétion 2, p. 218 et un exercice d'identification 3 p.219 portent sur les 
connecteurs temporels (puis, d'abord, enfin, lorsque, pendant que, avant que) et se réfèrent donc à la grammaire énonciative.

Dans l'exercice 1 p.218, on propose de conjuguer convenablement les verbes entre parenthèses pour décrire Jean Valjean. Les temps ciblés sont les temps du récit. Il s'agit de grammaire textuelle.

\section{Remarques concernant la grammaire dans les manuels}

Nous remarquons la récurrence de certains points de grammaire proposés dans les manuels des trois niveaux. La progression est donc en spirale.

Ainsi « le présent intemporel » proposé dans le projet 1 de première année (Campagne d'information) est repris dans le projet 1 de $3^{\text {ème }}$ AS (Dans le cadre de la commémoration d'une journée historique, réaliser une recherche documentaire). « La tournure impersonnelle » étudiée aussi dans le projet $1 \mathrm{du}$ manuel de $1^{\text {ère }} \mathrm{AS}$ est un point de langue dans le projet 3 du manuel de $2^{\text {ème }}$ AS (Procès pour défendre les valeurs humanistes). Les formes de "L'interrogation» sont également analysées en $1^{\text {ère }}$ AS (Projet1) et dans le cadre du projet 3 (Mettre en scène un spectacle) dans le manuel de $2^{\text {ème }}$ AS. $\mathrm{La}$ «nominalisation » est décrite en $1{ }^{\text {ère }} \mathrm{AS}$ (dans le fait divers « projet 3 ») puis en $3^{\text {ème }}$ AS (dans des textes d'Histoire «projet1»). Des exercices sur l'emploi du conditionnel sont proposés dans le manuel de $1^{\text {ère }}$ AS (projet 3) et celui de $2^{\text {ème }}$ AS (projet1).

«Les substituts grammaticaux » aussi font l'objet d'exercices dans le projet 1 du livre de $1^{\text {ère }}$ AS et le projet 3 du livre de $3^{\text {ème }}$ AS.

Des exercices portant sur le discours rapporté sont donnés dans le cadre de «L'interview» (projet 1 de $1^{\text {ère }} \mathrm{AS}$ ) et dans les textes de théâtre (projet 2 de $2^{\text {ème }}$ AS). Des exercices sur « la relative » existent en $1^{\text {ère }}$ AS (projet 1) et en $2^{\text {ème }}$ AS (projet 4).

Enfin, des exercices établissent les relations logiques entre les phrases dans les trois manuels (projet 1 de $1^{\text {ère }} \mathrm{AS}$, projet 1et projet 3 de $2^{\text {ème }} \mathrm{AS}$, et projet 2 de $3^{\text {ème }} \mathrm{AS}$ ).

Ceux-ci proposent des exercices de transformation, d'identification, de complétion, de réemploi, de classement. Les trois premiers types d'exercices sont les plus nombreux.

Ainsi, par exemple pour les exercices de transformation dans le manuel de $1^{\text {ire }}$ AS dans l'exercice 5 page 65 , dans le cadre du point de grammaire « La tournure impersonnelle », il est demandé aux élèves de transformer des phrases pour obtenir des constructions impersonnelles.

Dans le livre de $2^{\text {ème }}$ AS, pour l'étude du gérondif, on demande dans l'exercice page 46 de transformer des propositions en utilisant des verbes au gérondif.

Dans le manuel de $3^{\text {ème }}$ AS à la page 61 , l'exercice 1 propose des transformations de phrases verbales en phrases nominales. 


\section{La place de la grammaire dans l'enseignement du français langue \\ étrangère dans le cycle secondaire en Algérie}

Pour ce qui est des exercices d'identification, on peut citer l'exercice 1 page $73 \mathrm{du}$ manuel de $2^{\text {ème }}$ AS qui demande l'identification des connecteurs logiques. (Il s'agit d'entourer les connecteurs et de nommer les relations logiques qu'ils introduisent). On retrouve pratiquement la même consigne dans le livre de $3^{\text {ème }} \mathrm{AS}$, les exercices 1 et 2 pages 116 (étude des rapports logiques).

Enfin, les exercices de complétion sont nombreux dans les 3 manuels.

Dans le livre de $1^{\text {ère }}$ AS, on demande de compléter les phrases par des modalisateurs proposés dans une liste (ex 7 p.120).

L'exercice 3 p.73 du manuel de $2^{\text {ème }}$ année propose aux apprenants de compléter des phrases en respectant une consigne (rapport logique donné), ceci dans le cadre de l'étude des relations logiques explicites.

Dans le manuel de $3^{\text {ème }} \mathrm{AS}$, pour étudier les formes d'injonction (projet 3 ) on demande dans l'exercice 9 p.170 de compléter des textes en choisissant des verbes performatifs proposés dans une liste.

Les théories linguistiques auxquelles se référent les exercices des trois manuels sont surtout la grammaire transformationnelle, la grammaire textuelle, la grammaire énonciative et la pragmatique.

Il faut aussi remarquer que tous les exercices de grammaire proposés dans les 3 manuels portent sur des points en rapport avec l'objectif du projet.

Ainsi, en $1^{\text {ère }} \mathrm{AS}$, des exercices sur l'impératif et le discours rapporté sont proposés dans le cadre de l'interview (projet1).

Dans le manuel de $2^{\text {ème }} \mathrm{AS}$, les exercices portant sur les rapports logiques sont en relation avec le plaidoyer et le réquisitoire du projet 3 .

De même, les exercices en relation avec l'injonction dans le manuel de $3^{\text {ème }}$ AS sont en rapport avec l'appel du projet 3 .

Ce qu'il faut retenir de cette analyse des trois manuels du secondaire est qu'il n'y a pas de leçons de grammaire mais des exercices de grammaire, que ceuxci sont en relation avec le projet, permettent et facilitent donc la réalisation de ce dernier.

Il faut noter également qu'il s'agit essentiellement d'exercices de transformation, d'identification et de complétion et que les théories sousjacentes sont surtout la grammaire transformationnelle, la grammaire textuelle et la grammaire énonciative.

\section{Conclusion}

Nous confirmons donc notre hypothèse: dans le cycle secondaire, la grammaire n'occupe pas une place prépondérante. Elle figure sous forme d'exercices en $1^{\text {ère }}$ et $3^{\text {ème }}$ année secondaire et quelques rappels de points grammaticaux (des encadrés) en $2^{\text {ème }}$ année secondaire. Mais nous pouvons aussi penser que l'enseignement de la grammaire a été effectué d'une manière systématique dans les deux cycles primaire et moyen et que dans le cycle secondaire, il est consacré à l'application de des normes grammaticales pour faciliter la compréhension et surtout l'expression écrite. Une heure de 
grammaire en moyenne est prévue pour chaque séquence sous forme de rappels et d'exercices. Dans cette optique, cela peut paraître suffisant.

Les trois manuels font référence aussi bien à la grammaire textuelle et énonciative qu'à la grammaire transformationnelle. Le professeur D. Hanachi avait déjà mentionné dans son travail de recherche sur l'adverbe la diversité des théories utilisées dans les manuels scolaires.

Cette hésitation entre grammaire implicite et grammaire explicite, ce mélange de théories sous-jacentes aux leçons de grammaire présentées, cette quasiabsence de la grammaire dans le cycle secondaire peuvent expliquer partiellement les difficultés auxquelles se heurtent les apprenants de $3^{\text {ème }}$ AS qui ne maîtrisent pas la grammaire en fin de formation après un parcours de 10 ans d'apprentissage.

\section{Bibliographie}

Cuq, J.P. (2003). Dictionnaire de didactique du français langue étrangère et seconde. Paris : Clé international.

Damar, M-E., (2009), Pour une linguistique applicable, l'exemple du subjonctif en FLE, Bruxelles, PIE - Peter Lang.

Galisson, R \& Coste, D., (1976). Dictionnaire de didactique des langues. Hachette.

Hannachi, D. (1993), L'adverbe dans les manuels scolaires : analyse syntaxique et didactique, Mémoire de magister, Institut des langues vivantes et étrangères, Université de Constantine.

Narcy-Combes, M.F., (2005), Précis de didactique. Devenir professeur de français. Ellipes Editions Marketing SA.

Robert, J.P. (2002). Dictionnaire pratique de didactique du FLE. Ophrys.

Documents officiels et manuels scolaires

Djilali KeltoumIEF, (2009). , Guide du professeur. Première année secondaire. ONPS.

Commission nationale des programmes, (2009). , Document d'accompagnement. Projet de programme. Français $1^{\text {ère }}$ AS. ONPS.

Djilali KeltoumIEF. (2009)., Guide du professeur. Deuxième année secondaire. ONPS.

Commission nationale des programmes, (2009). , Document d'accompagnement. Projet de programme. Français 2ème AS. ONPS.

Fathi MahboubilEF. (2009)., Guide du professeur. Troisième année secondaire. ONPS.

Commission nationale des programmes, (2009). , Document

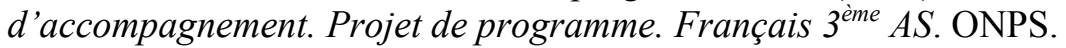

Djilali Keltoum IEF, Amal Boultif PES \&AllelLefsih PES (2009), Première année secondaire Français. ONPS. 
La place de la grammaire dans l'enseignement du français langue étrangère dans le cycle secondaire en Algérie

BaroudiZegrar IEF, Abdelkader Boumous IEF \& Rachida Betaouf (2011), Deuxième année secondaire Français. ONPS.

FethiMahboubi IEF, Mohamed Rekkab IEEM, AzzeddineAllaoui IEEM (2011), Troisième année secondaire Français. ONPS. 